\title{
Introducing BAX: A database for X-ray clusters and groups of galaxies ${ }^{\star}$
}

\author{
R. Sadat ${ }^{1}$, A. Blanchard ${ }^{1}$, J.-P. Kneib ${ }^{1,3}$, G. Mathez $^{1}$, B. Madore ${ }^{2}$, and J. M. Mazzarella ${ }^{2}$ \\ ${ }^{1}$ Laboratoire d'Astrophysique de l'Observatoire Midi-Pyrénées, 14 avenue E. Belin, 31400 Toulouse, France \\ e-mail: rsadat@ast.obs-mip.fr \\ 2 California Institute of Technology, Jet Propulsion Laboratory, MS 100-22, Pasadena, CA 91125, USA \\ 3 Astronomy Department, California Institute of Technology, Pasadena, CA 91125, USA
}

Received 1 July 2003 / Accepted 19 April 2004

\begin{abstract}
We present BAX, Base de Données Amas de Galaxies X (http://bax.ast.obs-mip.fr), a multi-wavelength database dedicated to X-ray clusters and groups of galaxies allowing detailed information retrieval. BAX is designed to support astronomical research by providing access to published measurements of the main physical quantities and to the related bibliographic references: basic data stored in the database are cluster/group identifiers, equatorial coordinates, redshift, flux, X-ray luminosity (in the ROSAT band) and temperature, and links to additional linked parameters (in X-rays, such as spatial profile parameters, as well as SZ parameters of the hot gas, lensing measurements, and data at other wavelengths, such as optical and radio). The clusters and groups in BAX can be queried by the basic parameters as well as the linked parameters or combinations of these. We expect BAX to become an important tool for the astronomical community. BAX will optimize various aspects of the scientific analysis of X-ray clusters and groups of galaxies, from proposal planning to data collection, interpretation and publication, from both ground based facilities like MEGACAM (CFHT), VIRMOS (VLT) and space missions like XMM-Newton, Chandra and Planck.
\end{abstract}

Key words. galaxies: clusters: general - X-rays: galaxies - astronomical databases: miscellaneous

\section{Introduction}

Clusters of galaxies are the largest virialized systems in the Universe and hence are thought to be useful probes of the universe. Historically, they have played an important role in cosmology, as they provided the first evidence of the presence of substantial amounts of dark matter in the Universe (Zwicky 1933). During the 70's, it has been discovered that galaxy clusters are strong X-ray emitters and it has been realized that crucial information can be obtained from the X-ray data and several space missions have been devoted to X-ray observations of clusters of galaxies (HEAO-1, 2, Ariel V, Einstein, EXOSAT, Ginga, Tenma, ROSAT, ASCA) and more recently XMM-Newton and Chandra. X-ray observations of clusters of galaxies have increased rapidly in the last years with the advent of good quality imaging and spectroscopy. All these observations resulted in large amount of X-ray data for thousands groups and clusters of galaxies published in an exponentially increasing number of papers.

The study of the statistical properties of X-ray clusters and groups and their evolution with redshift (i.e. their

* Database is only available in electronic form at http://bax.ast.obs-mip.fr and at the CDS via anonymous ftp to cdsarc.u-strasbg.fr $(130.79 .128 .5)$ or via

http:s//cdsweb.u-strasbg.fr/cgi-bin/qcat?J/A+A/424/1097 temperature and luminosity functions, as well as their luminosity-temperature relation) allow to constrain the cosmological parameters and to place strong constraints on large scale structure theories (Peebles et al. 1989; Oukbir \& Blanchard 1992; Hattori \& Matsuzawa 1995; Eke et al. 1996; see Rosati et al. 2002, for a review). The X-ray properties of groups and clusters indicate important effects of non-gravitational processes (Kaiser 1991; Evrard \& Henry 1991), which have fundamental consequences on the theory of galaxy formation. The study of chemical abundances in the intracluster cluster medium and its evolution should provide us with valuable information on metal enrichment of the universe and the history of nucleosynthesis in the universe and therefore on the star formation history (Mushotzky \& Lowenstein 1997).

Moreover clusters of galaxies are extended and often complex objects that can be observed at several wavelengths, leading to an increasing amount of scientific data and publications. However, access to this information through World Wide Web (WWW) is limited. Although some information (catalogs, tables, bibliographic references...) can be retrieved from existing databases such as NED (Mazzarella et al. 2001), SIMBAD (Wenger et al. 2000) or VIZIER (Ochsenbein et al. 2000), more specific information on X-ray clusters properties cannot presently be easily obtained. 
We attempt to provide an easy X-ray cluster parameter retrieval, by introducing the BAX (Base de données d'Amas de galaxies X) X-ray cluster online database, which is designed to support scientists, space missions and ground based observatories in the planning, interpretation and publication of research on galaxy clusters. Such a database is strongly needed and should be particularly useful to the astronomical community for the analysis and understanding of the global properties of groups and clusters and the correlations between their physical properties.

\section{Main objectives}

The primary goal of BAX is to help scientists working on X-ray clusters and groups, in their activity from proposal planning to data collection, reduction, interpretation and publication, by allowing a rapid and efficient access to the ensemble of published data measurements and bibliographical references on existing $\mathrm{X}$-ray galaxy clusters and groups, through a comprehensive and an easy-to-use tool. In its first implementation, BAX therefore:

- allows access to all published basic data and the corresponding bibliographic references;

- allows data collection and sampling through various selection criteria;

- helps in preparing proposals for future X-ray observations.

In a second stage, BAX will provide users with new capabilities that will be defined on the basis of their needs. These future improvements are described in Sect. 5.

\section{How does BAX operate?}

For a given cluster or group, BAX contains:

- the J2000 equatorial coordinates $(\alpha, \delta)$ and redshifts $z$, generated automatically from the NASA/IPAC Extragalactic Database (NED);

- a set of basic X-ray measurements: $\mathrm{X}$-ray fluxes $\left(F_{\mathrm{X}}\right)$ in ROSAT band, $\mathrm{X}$-ray luminosities $\left(L_{\mathrm{X}}\right)$ converted in the ROSAT [0.1-2.4 keV] band, and the X-ray temperatures $\left(T_{\mathrm{X}}\right)$;

- the corresponding bibliographical references.

Depending on the menu query, the user can search for an individual cluster by name or for an ensemble of clusters that meet chosen selection criteria, such as equatorial coordinates, redshifts, cluster basic data $\left(F_{\mathrm{X}}\right.$, $L_{\mathrm{X}}, T_{\mathrm{X}}$ ) or on linked parameters (see the help page http://bax .ast.obs-mip.fr/bax-help.html for the list of such linked parameters).

\subsection{Query functions through the Web interface}

BAX is accessible through the WWW interface located at http://bax.ast.obs-mip. fr site. The user starts his search through the main menu. By now five query modes are proposed: By name, By positions, By parameters, By keywords and Multi-criteria, this latter menu allows the combination of all the modes. BAX will then retrieve data and/or bibliographic references on a given cluster or on a list of clusters that respond to the chosen criteria. The outputs are:

- the cluster acronyms;

- the equatorial J2000 coordinates $(\alpha, \delta)$;

- the cluster redshift $z$;

- the canonical measurement of the three basic data $F_{\mathrm{X}}, L_{\mathrm{X}}$ and $T_{\mathrm{X}}$.

The canonical measurements are selected among recent and accurate existing measurements, and $F_{\mathrm{X}}$ and $L_{\mathrm{X}}$ are homogenized by conversion to the ROSAT [0.1-2.4 keV] energy band. The user can ask for further information to get access to all published measurements of the basic data through the all measurements query and/or access to an ensemble of bibliographic references selected by keywords criteria through the Bibliography criteria. The bibliography search will retrieve the papers associated to at least one of the chosen keywords.

The heart of BAX is the database with its Web interface query service, but BAX is also conceived to provide the user with various tools to ease and optimize the use of the database as well as to allow data analysis. For instance, BAX contains the coordinate conversion and precession and the flux converter that allows to convert fluxes from any given energy band to the [0.1-2.4 keV] ROSAT reference band.

\subsection{Establishing the Temperature-Luminosity relation using the "By multi-criteria" query}

In the following we will illustrate an exemple of BAX capabilities through the "multi-criteria" query. As already mentionned, this menu allows a more elaborated query by combining criteria on name, positions, physical parameters and keywords. With this feature one can easily build up a sample of galaxy clusters selected on a set of criteria, (useful for statistical studies). An example of query is to build a sample of clusters with available luminosity and temperature measurements, in order to study one of the best studied correlations among X-ray clusters the Luminosity-Temperature relation (Mitchell et al. 1979; Edge \& Stewart 1991; David et al. 1993; Arnaud \& Evrard 1999). This relation between the X-ray temperature $T_{\mathrm{X}}$ and luminosity $L_{\mathrm{X}}$ is important because it is indicative of the internal structure in clusters of galaxies and possible variations in the gas mass fractions (the ratio of the X-ray gas to total mass) since the luminosity is determined by the mass of the intra-cluster medium emitting the hot X-ray gas and the temperature is determined by the total gravitating mass. The user can retrieve such a sample by simply filling the adequate fields in the multi-criteria menu form (here the $L_{\mathrm{X}}$ and $T_{\mathrm{X}}$ fields). BAX will return a list of clusters that meet all the criteria. For each cluster of the sample BAX provides with the basic data $\alpha, \delta, z, F_{\mathrm{X}}, L_{\mathrm{X}}$ and $T_{\mathrm{X}}$ Here again further information can be obtained for each single cluster of the list through the all published measurements and/or the bibliography. Figure 1 presents the resulting $L_{\mathrm{X}}-T_{\mathrm{X}}$ relation we derived from the luminosity and temperature measurements we found using BAX multi-criteria query. 


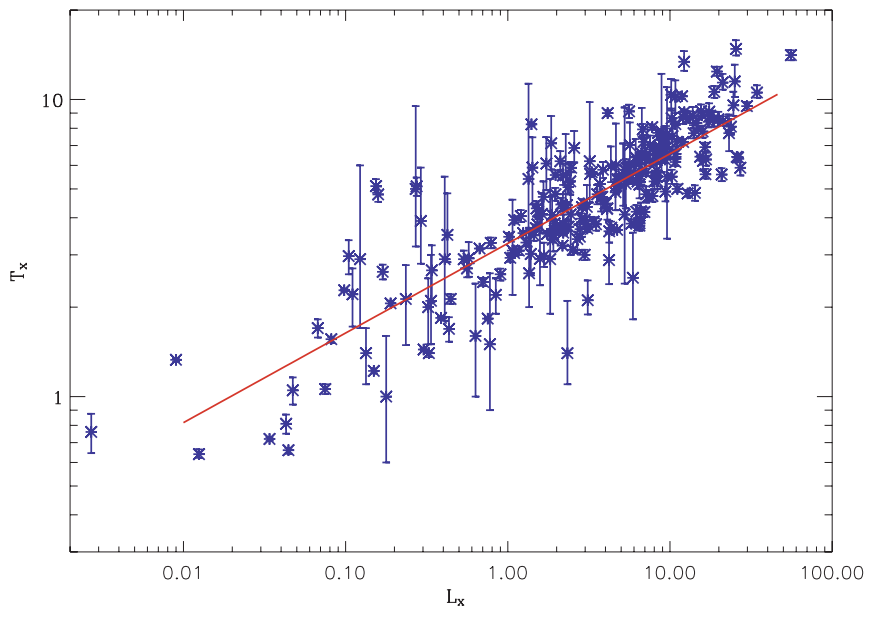

Fig. 1. The $L_{\mathrm{X}}-T_{\mathrm{X}}$ relation as obtained using the 247 clusters with measured $L_{\mathrm{X}}$ and $T_{\mathrm{X}}$ found in BAX database. The (red) solid line is the result of fitting $T_{\mathrm{X}}$ versus $L_{\mathrm{X}}$ to the data. We find a correlation slope of 3.3. Note here that luminosities we use are luminosities in the ROSAT [0.1-2.4] rest-frame band.

\section{Current status}

The number of published papers on X-ray clusters and groups is increasing rapidly, and this rise should hold in the forthcoming years because of existing and future space and ground facilities. Updating the database is therefore a long-term task that is necessary for its scientific relevance. By now, the most important large X-ray cluster catalogs have been included in the database, except for catalogs that are not yet published such as the MAssive Cluster Survey (MACS, Ebeling et al. 2001), the ROSAT-ESO flux limited X-ray (REFLEX) cluster survey (Böhringer et al. 2001) and the ROSAT Deep Cluster Survey (RDCS, Rosati et al. 1995, 1998). BAX is currently kept up-to-date on a daily basis by integrating the most recent bibliographic references mainly resulting from Chandra and XMM-Newton observations very soon after their publication in journals referenced in ADS. At the time of writing, BAX is complete in terms of XMM-Newton publications and published papers during year 2003 and it contains data on 1579 clusters and groups, with 8116 measurements and 33185 linked keywords, among which 1371 clusters and groups with available flux measurements in BAX and 298 with temperature measurements. The published version of the database was released in September 2002. The "debugged" version of the database is running since May 2003. 7949 connections and 3938 queries have been recorded since that date. During the year 2003, BAX recorded roughly 300-400 connections per month with approximately the same number of queries, and these rates are increasing in 2004.

\section{BAX updates and future improvements}

BAX has been conceived to be adaptable: for example, the list of linked keywords can be easily modified by the administrator and linked parameters can be turned into basic parameters stored in the database. Beyond existing data on galaxy clusters and groups, BAX will evolve to offer new functionalities accordingly to the needs of users. For instance, among various requests that were asked for are:

- the direct access to other existing X-ray archives;

- the access to properties of virtual clusters obtained from numerical simulations;

- the addition of dynamical links to other data services;

- the extension to non X-ray galaxy clusters and groups: optical, and radio parameters, as well as gravitational lensing and SZ parameters;

- the implementation of new tools such as: XMM-Newton data analysis using the SAS software, tools to visualize the query outputs (histograms, charts);

- the development of a portal for public outreach.

The extension to non X-ray observations is motivated by several large scale facilities, either ongoing or scheduled in the future, which will provide us with massive sets of data concerning known clusters and groups, as well as the discovery of numerous new such systems: large scale optical surveys (e.g. SDSS, CFHT Legacy Survey, GOODS), will produce new catalogs of optically selected clusters, at the same time weak lensing surveys from ground-based (e.g. CFHT Legacy Survey) or space missions are going to provide direct lensing detection of dark matter concentrations, perhaps pointing to dark groups and clusters (Erben et al. 2000). In the near future, the Planck Surveyor and ground-based telescopes such as APEX, SPT, ACT should produce large catalogs of SZ detected clusters (Barbosa et al. 1996; Carlstrom et al. 2002). In this context, BAX will be extremely useful at several levels: in its present version, it will allow to obtain the cross identification with known X-ray clusters, but also it will ease the statistical correlation of the survey clusters according to various physical properties. Finally, BAX will be one of the privileged ways to facilitate the distribution of survey results.

At a more sophisticated level, BAX might provide the user with tools for quick look data product from ongoing missions. It is also intended to plug as an on-line web server tool an automated pipeline (described in Marty 2003) to reduce EPIC MOS \& PN spectro-imaging data. More general software tools for X-ray data analysis, simulation and proposal preparation tools will also be available as a service provided by BAX, by using public on-line version of existing tools such as WebPIMMS, XANADU, FTOOLS and other HEASARC (High Energy Astrophysics Science Archive Research Center) software tools.

\section{BAX in the context of a global virtual observatory}

Among the purposes of the Virtual Observatory (VO) is the interconnection and federation of data archives, surveys and software tools from observatories using common database query standards and data transfer protocols. The need of such a VO is driven by the growing size of astronomical data sets due to new large facilities and the ambition to discover new scientific results from connected large multi-wavelength data sets using data mining tools. In this context, BAX is able to provide 
its specific contribution to the construction of the global virtual observatory, including the bibliographic aspect. We expect that the future developments, such as the extension to multiwavelength published data on galaxy clusters and groups, the implementation of on-line data analysis and simulation software tools and the interoperability with other archival services will allow to specify in more details the role that BAX could play in the Virtual Observatory.

\section{Brief summary}

BAX is an online database of X-ray clusters and groups of galaxies designed to support scientists at different steps of their research from proposal planning to data collection, interpretation and publication. BAX provides published data measurements of the basic properties of clusters and groups, including pointers to related bibliographical references. The ultimate goal of BAX is to provide not only an interface to query its database but also to serve as a portal for the general community of scientists working on clusters and groups of galaxies. We expect BAX to become an essential tool for the astronomical community especially in view of preparing missions like Planck Surveyor, which will detect up to 50000 individual galaxy clusters through the SZ effect on the cosmic microwave background (Kay et al. 2001). In the future, we hope to develop new capabilities and services by developing new collaborations with other organizations such as the CDS (Strasbourg), NED (Caltech), HEASARC (NASA), LEDAS (Leicester), as well as with the XMM-Newton and Chandra X-Ray observatories, in view of contributing to the construction of the Virtual Observatory.

Acknowledgements. BAX has been supported by the Centre National d'Études Spatiales (CNES), the french Programme National de Cosmologie (PNC) and the Observatoire de Midi-Pyrénées (OMP). We thank S. Drake and the HEASARC team for the announcement on BAX in the HEASARC Home Page. We also would like to thank G. Mamon for reading the paper and for useful discussions.

\section{References}

Arnaud, M., \& Evrard, A. E. 1999, MNRAS, 305, 631

Barbosa, D., Bartlett, J. G., Blanchard, A., \& Oukbir, J. 1996, A\&A, 314,13

Böhringer, H., Voges, W., Huchra, J. P., et al. 2001, A\&A, 369, 826

Carlstrom, J. E. L., Holder, G. P., \& Reese, E. D. 2002, ARA\&A, 40, 643

David, L. P., Slyz, A., Jones, C., et al. 1993, ApJ, 412, 479

Eke, V. R., Cole, S., Frenk, C. S., \& Henry, J. P. 1996, MNRAS 298, 1145

Ebeling, H., Edge, A. C., \& Henry, J. P. 2001, ApJ, 553, 668

Edge, A. C., \& Stewart, G. C. 1991, MNRAS, 252, 414

Erben, T., van Waerbeke, L., Mellier, Y., et al. 2000, A\&A, 355, 23

Evrard, A. E., \& Henry, J. P. 1991, ApJ, 383, 95

Hattori, M., \& Matsuzawa, H. 1995, A\&A, 300, 637

Kaiser, N. 1991, ApJ, 383, 104

Kay, S. T., Liddle, A. R., \& Thomas, P. A. 2001, MNRAS, 325, 835

Kurtz, M. J., Eichhorn, G., Accomazzi, A., et al. 2000, A\&AS, 143, 41

Marty, P. 2003, Ph.D. Thesis

Mazzarella, J. M., Madore, B. F., \& Helou, G. 2001, SPIE, 4477, 20

Mushotzky, R. F., \& Lowenstein, M. 1997, ApJ, 481, L63

Mitchell, R. J., Dickens, R. J., Burnell, S. J. B., Culhane, J. L. 1979, MNRAS, 189, 329

Ochsenbein, F., Bauer, P., \& Marcout, J. 2000, A\&AS, 143, 23

Oukbir, J., \& Blanchard, A. 1992, A\&A, 262, L21

Peebles, P. J. E., Daly, R. A., \& Juszkiewicz, R. 1989, ApJ, 347, 563

Raymond, J. C., \& Smith, B. W. 1977, ApJS, 35, 419

Rosati, P., Borgani, S., \& Norman, C. 2002, ARA\&A, 40, 539

Wenger, M., Ochsenbein, F., Egret, D., et al. 2000, A\&AS, 143, 9

Zwicky, F. 1933, Helvetica Phys. Acta, 6, 110 